\title{
Psychokardiologie - Psychosomatik im Herzen der Medizin
}

$\mathrm{H}$ erz-Kreislauferkrankungen stellen die häufigste Todesursache in Deutschland dar. Weltweit wird allein die koronare Herzkrankheit im Jahre 2020 voraussichtlich die führende Ursache krankheitsbedingter Beeinträchtigungen sein - direkt gefolgt von der unipolaren Major Depression. Die Komorbidität dieser beiden Erkrankungen stellt allein angesichts ihre quantitativen Bedeutung schon eine enorme Herausforderung für die Medizin dar und bildet auch einen Roten Faden des vorliegenden Heftes.

Gerade die Depression interagiert aber auch, wie im Beitrag von Buss sorgfältig herausgearbeitet, auf vielfältige Weise mit der koronaren Herzkrankheit und ihrem Verlauf. Ihre Erkennung und Behandlung wird daher von aktuellen kardiologischen Leitlinien gefordert, über die der Beitrag von Albus einen sehr kompetenten Überblick gibt.

Ungewiss ist, ob sich damit der lang gehegte Wunsch der Psycho-Disziplinen erfüllt, mit einer psychotherapeutischen oder psychopharmakologischen Intervention den somatischen Verlauf einer organischen Erkrankung günstig beeinflussen und eventuell sogar das Leben verlängern zu können. Dies wäre natürlich ein bestechender Beleg für die auch kausale Bedeutung der Depression für kardiale Erkrankungen.

Andererseits wäre es bedenklich, die Indikation für die Behandlung psychischer Störungen überwiegend mit ihrem somatischen Nutzen zu begründen. Psychische Störungen rechtfertigen, ja verlangen, um ihrer selbst willen eine kunstgerechte Behandlung: Sie führen zu massiven Beeinträchtigungen der Lebensqualität der betroffenen Patienten und ihrer sozialen Umwelt einschließlich der Arzt-Patient-Beziehung und verursachen hohe Kosten durch inadäquate Inanspruchnahme somatisch-medizinischer Leistungen. Ihre Behandlung führt zu einer gut belegten symptomatischen Besserung und einem deutlichen Anstieg der Lebensqualität. Davon kann die somatische Behandlung durch bessere Adhärenz der Patienten sowie Vermeidung somatisch unbegründeter Diagnostik und z.T. invasiver - Therapien nur profitieren.
Die moderne Kardiologie verfügt über ein reiches Repertoire nachgewiesen wirksamer Behandlungen, die aber, wie Jünger et al. in ihrer Übersicht zur Psychosomatik der Herzinsuffizienz berichten, nur sehr unvollständig von den Patienten wahrgenommen werden. Grundsätzlich hat sie aber sicher das Potenzial, Leben zu verlängern. Demgegenüber sind die Effekte vieler kardiologischer Behandlungen auf die Lebensqualität vergleichsweise ernüchternd. Hier liegt klar eine Domäne der sprechenden Medizin.

Diese in die kardiologische Routine zu integrieren, ist eine der großen Aufgaben der Zeit. Dafür braucht es konkrete Modelle, etwa das hier von Fritzsche aufgrund langjähriger Erfahrungen dargestellte Liaisonmodell, das sehr konkret und praxisnah einen Vorschlag für den psychosomatischen Umgang mit Herzpatienten in der Akutversorgung beschreibt.

Damit können die Patienten sich ganzheitlich mit ihren körperlichen und psychischen Krankheitsaspekten angenommen und behandelt fühlen. Zugleich wird die Aufspaltung in Organmedizin und Seelenmedizin überwunden und das kardiologische „add years to life“ durch eine gezielte Einbeziehung des Subjektiven, das „add life to years", ergänzt.

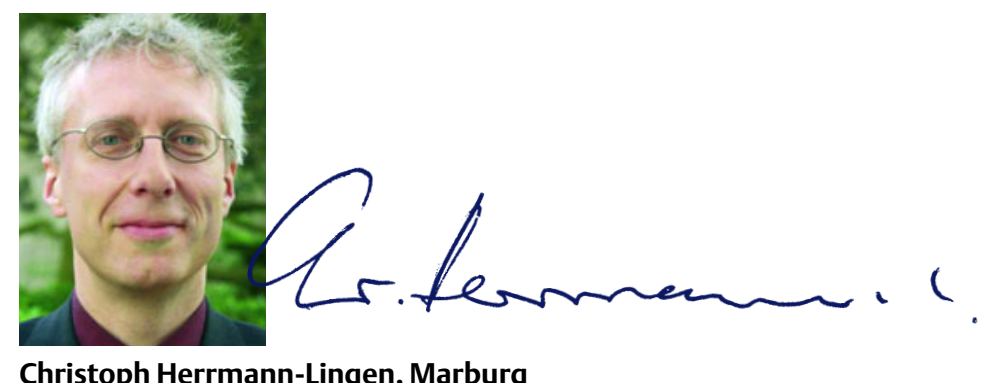

Christoph Herrmann-Lingen, Marburg 